\title{
Continuous non-invasive monitoring of the skin temperature of HSCT recipients
}

\author{
Maarten van Vliet • J. Peter Donnelly • \\ Carin M. J. Potting • Nicole M. A. Blijlevens
}

Received: 11 February 2009 /Accepted: 22 March 2009/Published online: 25 April 2009

(C) The Author(s) 2009. This article is published with open access at Springerlink.com

\begin{abstract}
Goals of work Empirical antibiotic therapy usually started in patients who are neutropenic following treatment with cytostatic chemotherapy for a haematological malignancy as soon as fever develops to forestall fulminant sepsis. Hence, accurate and timely detection of fever is crucial to the successful management of infectious complications in these patients. We report an investigation of the feasibility and validity of continuous non-invasive body temperature measurement.

Patients and methods The feasibility of non-invasive continuous measurement of the skin temperature was investigated using the Propaq ${ }^{\circledR}$ device in a cohort of 33 patients receiving an allogeneic HSCT who were all at risk of developing a febrile episode. Non-invasive continuous measurement of the skin temperature (CST) was compared with a standard episodic axillary temperature measurement (EAT) five times daily using a Terumo ${ }^{\circledR}$ device. The study period entailed monitoring during the 10 or 12 days that profound neutropenia was expected to be present.

Main results Measuring the skin temperature continuously and accurately by using the Propaq ${ }^{\circledR}$ was feasible. The CST correlated well with the EAT measurements (Pearson $r=$ 0.782). Compared to EAT, the start of empirical therapy could be started $2.5 \mathrm{~h}$ earlier when relying on continuous measurements than was possible with EAT.
\end{abstract}

M. van Vliet $(\bowtie) \cdot J$. P. Donnelly $\cdot$ C. M. J. Potting $\cdot$

N. M. A. Blijlevens

Department of Haematology,

Radboud University Nijmegen Medical Centre,

Geert Grooteplein 10, P. O. Box 9101, 492, 6500HB Nijmegen,

The Netherlands

e-mail: m.vanvliet@cisvpl.umcn.nl
Conclusion Continuous skin temperature measurements are feasible and valid compared to the conventional temperature measurement and may improve the management of infections by earlier detection of fever in neutropenic patients.

Keywords Cancer · Infection · Prompt antibiotic therapy · Neutropenia $\cdot$ Body temperature

\section{Introduction}

Approximately $90 \%$ of cycles of intensive chemotherapy used to treat acute leukaemia are complicated by prolonged and profound neutropenia that is usually accompanied by fever and infections [11]. Neutropenic patients who are febrile are at high risk for developing sepsis, which often necessitates acute admission to the Intensive Care Unit (ICU) and is associated with a high mortality [3]. Fever is usually the most obvious sign of a systemic inflammatory response [5], so measuring the body temperature is one of the most important nursing tasks. Instituting empirical broad-spectrum antimicrobial therapy promptly at the first sign of fever has been shown crucial to patient survival and remains the cornerstone of infection management $[2,8,13]$. Mortality associated with fever and neutropenia has dramatically decreased over the last three decades and is, in no small measure, the result of general adherence to the concept of giving empirical therapy with broad-spectrum antibiotics promptly at the onset of fever [7]. Needless to say this approach hinges upon the early detection fever by measuring the body temperature.

A variety of methods are used to measure body temperature, involving different sites, instruments and techniques. The core temperature is best measured in the 
pulmonary artery using a Swan-Ganz catheter [4, 15], but this is impossible to employ on a haematology ward in daily clinical practice. Mercury thermometers are now obsolete and have been replaced by electronic devices. These thermometers accurately predict temperature and can be adapted for use at different body sites to measure temperature orally, axillary, tympanically or rectally [14]. However, the choice of sites for adults on a haematology ward is limited. Temperature is most often measured orally although oral mucositis results in erroneous measurements [1]. Rectal measurement during thrombocytopenia is too dangerous because of the risk of rectal bleeding. There are convincing data to show that tympanic temperature measurement is reliable, but the results are more variable than found with rectal or oral readings [12]. For these reasons, we have continued to measure the axillary temperature for our adult population [10].

For continuous measurements, a temperature sensor connected to the Welch-Allyn ${ }^{\circledR}$ Propaq monitor was used. This device is able to measure temperature continuously. In this audit, $24 \mathrm{~h}$ non-invasive measurement of skin temperature was explored. The goals were:

1. To assess the feasibility of continuously monitoring the skin temperature of adults admitted to the haematology ward for an HSCT.

Table 1 Demographic data
2. To assess the validity of the data by calculating the correlation between the values measured by the continuous device and the axillary measurements.

\section{Patients and methods}

Patient population

The audit was carried out on the haematology ward of the Radboud University Nijmegen Medical Centre where 33 patients admitted to receive an HLA compatible allogeneic haematopoietic stem cell transplant (HSCT) from a sibling or a voluntary unrelated donor, consented verbally to participate. Ethics Committee approval was not required. Both myeloablative or non-myeloablative conditioning was used for prepatory regimen. Demographic data of the patients are shown in Table 1.

Procedure

Participating patients had their body temperature measured simultaneously by two different instruments. This period was defined as the first 10 days post-transplant or, in the case of idarubicin, 2 days before HSCT until 10 days post-

\begin{tabular}{ll}
\hline Gender & 14 Male \\
Age & 19 Female \\
Type of transplant & Mean 48.0 years (range 22-64) \\
& Sibling HSCT (17) \\
& Voluntary Unrelated Donor HSCT (16) \\
Underlying disease & Acute myeloid leukaemia (10) \\
& Myelodysplastic syndrome (5) \\
& Severe aplastic anaemia (4) \\
& Chronic lymphocytic leukaemia (3) \\
& Non-Hodgkin's lymphoma (4) \\
& Acute lymphocytic leukaemia (3) \\
& Multiple myeloma (1) \\
& Chronic myeloid leukaemia (1) \\
& Myelofibrosis (1) \\
& Acute basophile leukaemia (1) \\
& Myeloablative \\
& Idarubicin-cyclophosphamide-total body irradiation (TBI) (9) \\
& ATG-cyclophosphamide-TBI (11) \\
& ATG-cyclophosphamide-fludarabine (4) \\
& Idarubicine-busilvex-cyclophosphamide (1) \\
Nonmyeloablative \\
Cyclophosphamide-fludarabine-TBI (2) \\
Cyclophosphamide-TBI (3) \\
Cyclophosphamide and fludarabine (2) \\
Cyclophosphamide-TLI (1) \\
Conditioning regimen & \\
\hline & \\
& \\
& \\
&
\end{tabular}


transplant as this drug was given 2 weeks before transplant whilst others were given within a week. Temperature was scheduled to be registered five times daily at fixed times: 03:00, 08:00, 13:00, 18:00 and 22:00. The maximum interval between two measurements was $5 \mathrm{~h}$.

\section{Instruments}

\section{EAT}

We used a Terumo ${ }^{\circledR}$ axillary thermometer, type C202, to measure axillary temperature. The device was calibrated for the range of $32.0-42.0^{\circ} \mathrm{C}$, in steps of $0.5^{\circ}$, by means of a warm water bath. The maximum deviation after $90 \mathrm{~s}$ was $0.02^{\circ}$, as was internally validated by the technical service (data not shown). The Terumo thermometer predicts the axillary temperature after approximately $60 \mathrm{~s}$.

\section{CST}

Continuous measurement was achieved by using a temperature sensor connected to the Welch-Allyn ${ }^{\circledR}$ Propaq monitor. This device measures temperature continuously preferably in a body cavity that is least exposed to ambient air. Patients were asked to carry the sensor either in their groin or axilla, though the former was preferred as it is least influenced by the patient's movements.

\section{Supportive care}

Empirical therapy was started once when the axillary temperature as measured by episodic axillary temperature (EAT) was equal to, or exceeded, $38.5^{\circ} \mathrm{C}$. When continuously measured skin temperature (CST) indicated $\mathrm{T} \geq$ $38.3^{\circ} \mathrm{C}$ axillary measurement was performed to see if EAT confirmed this. At the onset of fever, blood was obtained for cultures, ceftazidime was administered empirically, and the attending physician was alerted. A blood culture was considered positive if one or more cultures yielded a microorganism, except for coagulase-negative staphylococci, for which two separate positive blood cultures yielding the same strain were required to be considered to represent bacteraemia [9].

\section{Audit}

Feasibility was tested in the first six patients, and a go/no-go decision to continue the audit was made. Carrying the device was found feasible when five of the six patients could bear the sensor during the day, and the monitor reflected the body temperature. Patients were interviewed to assess the feasibility of carrying a temperature device on the body during the day. The validity of the continuous measurements was assessed by comparing pairs of simultaneously measured values and calculating the correlation and regression between them.

Data analysis

Data on the validity of the measurements were collected by the nurses of the ward. A registration form was specially developed to record the data. All temperature measurements were recorded during 10 or 12 days of observation.

Data were imported in SPSS ${ }^{\circledR}$ version 14.0 for data analysis. Correlation and regression analysis were performed to assess validity of the experimental measurements. The outliers in temperature difference, defined as a $0.5^{\circ}$ difference $(\Delta T)$ or more between the two simultaneously used methods were analysed on possibly confounding factors age, BMI and gender.

\section{Results}

\section{Feasibility}

Feasibility was assessed after the first six patients had been observed, and the decision was made to continue when five patients had been shown to have no problems carrying the CST device. A total of 33 patients were included and 29 completed the observation period successfully. Each patient could move around freely as the sensor was not attached to their body and could easily be disconnected temporally when desired. During the audit, one patient was uncomfortable carrying the device during the day and withdrew from the study for that reason. Three patients complained about the sensor and considered abandoning the study but decided to continue. The CST did not reflect the body temperature in one case as the patient's physique did not permit sufficient isolation from ambient air.

\section{Validity of the CST related to the EAT}

Regarding the difference in the simultaneously measured body temperature between the EAT and the CST $(\Delta T)$, 1,409 values were valid for evaluation as missing values were not properly recorded on the registration form. As shown in Fig. 1, 90\% of the differences in measurements are located in a range of $-0.5^{\circ} \mathrm{C}$ to $0.9^{\circ} \mathrm{C}$. The CST measured both higher and lower temperatures than was recorded for EAT and frequency, the values of both were distributed in a Gaussian fashion though EAT was on average $0.23^{\circ}$ higher (Fig. 1).

There was a good correlation between the two methods (Fig. 2; Pearson correlation of $0.782, p \leq 0.01$ ). Regression analysis yielded an $R^{2}$ of 0.612 and the intercept and slope 


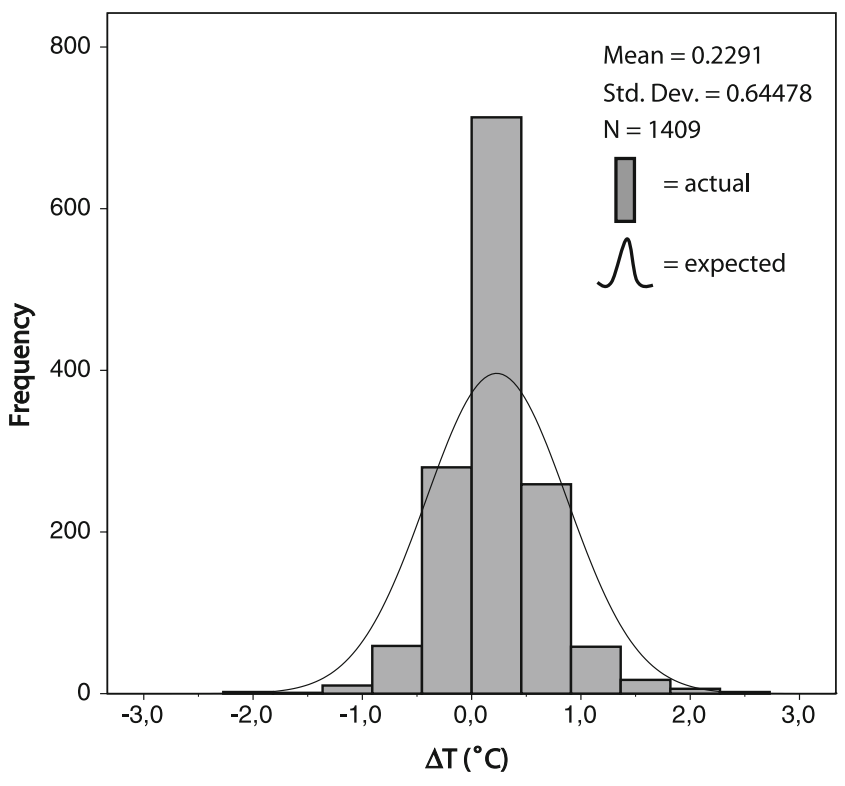

Fig. 1 Histogram on the difference (EAT minus CST) in simultaneously measured temperature by two different methods outliers are left out)

indicated that $\Delta T$ was most pronounced at higher values: $y=$ $0.934^{*} x+2.259$. For an axillary temperature of $36^{\circ} \mathrm{C}$, the mean deviation of the CST was $0.12^{\circ}$; for an axillary temperature of $40.5^{\circ} \mathrm{C}$, the mean deviation of the CST was $0.41^{\circ}$.

$\Delta T$ was 0.5 or more in $29.5 \%$ of all cases. Per patient, a $\Delta T \geq 0.5$ was found in $2 \%$ to $69 \%$ of the total number of measurements suggesting that patient characteristics played a considerable role. Analysis of 'gender' and ' $\Delta T \geq 0.5$ ' showed no significant difference between the groups $(p=$
$0.309)$, and there was no influence of either BMI $(p=0.227)$ or age $(p=0.614)$.

In $78 \%$ of patients, bacteraemia was detected during the initial febrile episode during neutropenia justifying the decision to start broad-spectrum antibiotic treatment.

Although it was not the primary object of the study, we noted that antibiotics were started over $2.5 \mathrm{~h}$ earlier for 12 out of 14 evaluable patients as a result of earlier detection of fever by continuous measurements.

\section{Discussion}

Fever is regarded as the most important means of identifying an inflammatory process in neutropenic patients, but there is reason for concern as the reliability of the common methods of temperature measurement all appears to have limitations. A high frequency of recording vital signs should be adopted with in every haematology ward's daily practice where early detection of fever can be lifesaving. Surprisingly, no published data were available on non-invasive continuous body temperature measurement in oncology patients.

The goals of this audit were to assess feasibility and validity of continuous skin temperature measurement (CST). This audit found that CST is feasible in clinical haematology patients and is valid compared to the traditional measurements except when a patient is physically incapable of carrying the sensor where it can remain sufficiently isolated.

The extreme values observed $(\Delta T \geq 0.5)$ were likely explained by the malpositioning of either of the instru-
Fig. 2 Scattergram with regression line on the correlation of two independent measurements of body temperature

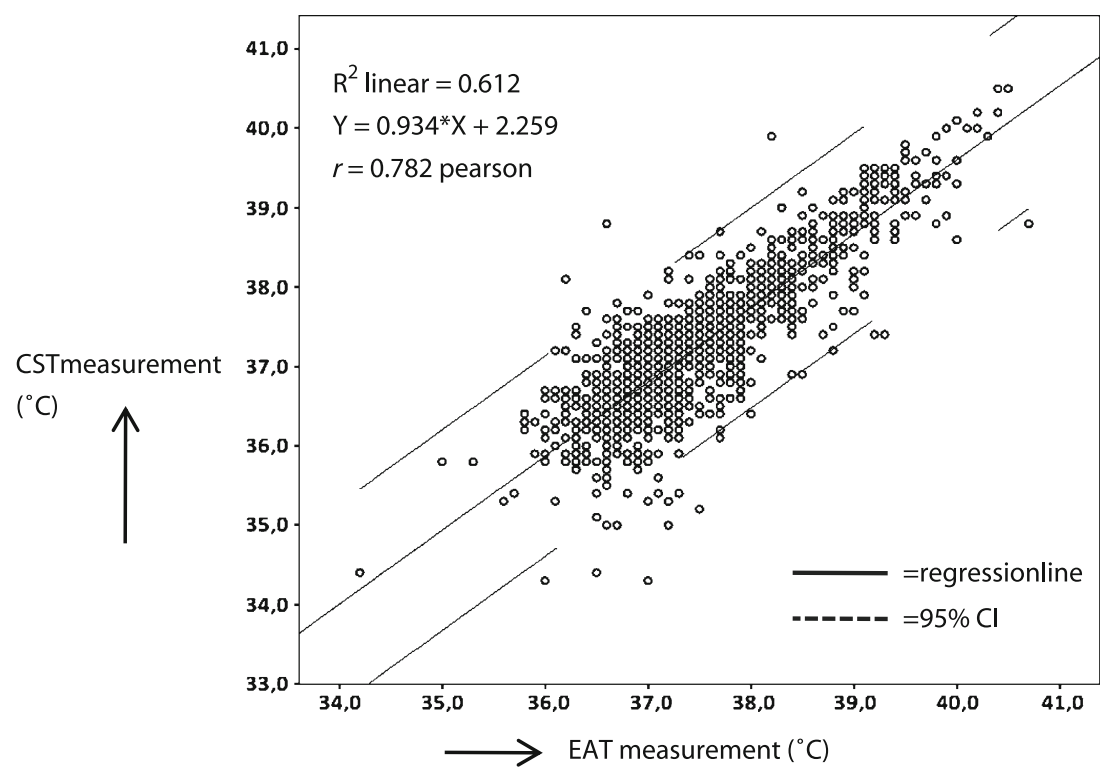


ments. The relationships between the distribution of $\Delta T \geq$ 0.5 and factors of the human physical composition such as gender, BMI and age were explored but these did not explain the findings. Both the standard instructions with the Terumo (proper placement and drying the axilla previous to the measurement) and the continuous device (isolation from outside air is necessary for reliable values) are sensitive to erroneous measurement. Also, the positioning of the device could account for this when done by the patients themselves. Alternatively this may reflect a common problem, namely inconsistency in the use of thermometry in daily practice.

Early detection of fever is a challenge during cycles of chemotherapy where the start of empirical antibiotic therapy can be life-saving during neutropenia. All guidelines emphasise the immediate start of empirical therapy at the onset of fever so it makes sense to increase the frequency of measurements to increase the chance that the onset of fever is detected promptly. This is not the case when the temperature is only measured every $4-6$ hours, but reliable continuous measurements allow the onset of fever to be caught in the act so to speak.

The fact that $78 \%$ of the patients actually had bacteraemia during the first episode of fever sufficiently justifies the start of broad-spectrum antibiotic treatment as soon as fever occurs. Hence, it could be argued that the lack of a rapid, reliable and continuous means of temperature monitoring may increase the risk for developing a systematic inflammatory response syndrome (SIRS), sepsis, shock and even death.

Although the concept of fever is familiar to nurse, the precise definition we should adopt for optimal patient outcomes is far from clear as a variety of thresholds varying between 37.5 and 38.5 are employed, and the temperature is measured in several different ways. For instance, the 2002 IDSA guidelines recommends using a definition of fever as a single measured oral temperature of $\geq 38.3$ or a

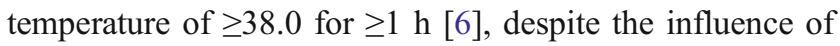
oral mucositis on oral thermometry [1]. Clearly, there is still much to be done.

\section{Conclusion}

Continuous temperature measurement makes it possible to monitor patients intensively during round the clock without involving extra personnel. Continuous body temperature measurement is commonly used in the ICU but is alien to haematology wards. Earlier detection of the onset of fever and SIRS makes earlier initiation of empirical therapy possible which might lead to fewer days with fever, reduce antibiotic usage, the length of stay and mortality and allow better deployment of nursing staff.
Future directions

Neither CST and EAT are considered to be the Gold standard for estimating the core temperature. Ideally, a valid means of continuously measuring temperature needs to be developed that is more reliably correlated to core temperature. However, it will be a challenge to find a method of reliably achieving this while being economic and userfriendly. In the meantime, further research should be done on a larger group of neutropenic patients exploring the consequences of earlier detection of fever on outcomes such as length of stay, costs and amount of antibiotics and survival.

Acknowledgement The author would like to thank Franka Dinnissen, the patients who volunteered and the nurses on the ward for their support and technical assistance.

Open Access This article is distributed under the terms of the Creative Commons Attribution Noncommercial License which permits any noncommercial use, distribution, and reproduction in any medium, provided the original author(s) and source are credited.

\section{References}

1. Ciuraru NB, Braunstein R, Sulkes A, Stemmer SM (2008) The influence of mucositis on oral thermometry: when fever may not reflect infection. Clin Infect Dis 46(12):1859-1863. doi:10.1086/ 588290

2. de Pauw BE, Deresinski SC, Feld R, Lane-Allman EF, Donnelly JP (1994) Ceftazidime compared with piperacillin and tobramycin for the empiric treatment of fever in neutropenic patients with cancer. A multicenter randomized trial. The Intercontinental Antimicrobial Study Group. Ann Intern Med 120(10):834-844

3. Dellinger RP, Levy MM, Carlet JM, Bion J, Parker MM, Jaeschke $R$ et al (2008) Surviving Sepsis Campaign: international guidelines for management of severe sepsis and septic shock: 2008. Crit Care Med 36(1):296-327

4. Fulbrook P (1993) Core temperature measurement in adults: a literature review. J Adv Nurs 18(9):1451-1460. doi:10.1046/ j.1365-2648.1993.18091451.x

5. Giuliano KK (2006) Continuous physiologic monitoring and the identification of sepsis: what is the evidence supporting current clinical practice? AACN Adv Crit Care 17(2):215-223. doi:10. 1097/00044067-200604000-00017

6. Hughes WT, Armstrong D, Bodey GP, Bow EJ, Brown AE, Calandra $T$ et al (2002) 2002 guidelines for the use of antimicrobial agents in neutropenic patients with cancer. Clin Infect Dis 34(6):730-751. doi:10.1086/339215

7. Klastersky J (2001) Empirical treatment of sepsis in neutropenic patients. Hosp Med 62(2):101-103

8. MacArthur RD, Miller M, Albertson T, Panacek E, Johnson D, Teoh L et al (2004) Adequacy of early empiric antibiotic treatment and survival in severe sepsis: experience from the MONARCS trial. Clin Infect Dis 38(2):284-288. doi:10.1086/379825

9. MacGregor RR, Beaty HN (1972) Evaluation of positive blood cultures. Guidelines for early differentiation of contaminated from valid positive cultures. Arch Intern Med 130(1):84-87. doi:10. 1001/archinte.130.1.84 
10. MacKenzie MA, van der Meer JW, van Heteren GM (1997) Ned Tijdschr Geneeskd 141(19):957-959 Clinical thermometry. II. Current dilemmas

11. Neuburger S, Maschmeyer G (2006) Update on management of infections in cancer and stem cell transplant patients. Ann Hematol 85(6):345-356. doi:10.1007/s00277-005-0048-2

12. Rabinowitz RP, Cookson ST, Wasserman SS, Mackowiak PA (1996) Effects of anatomic site, oral stimulation, and body position on estimates of body temperature. Arch Intern Med 156 (7):777-780. doi:10.1001/archinte.156.7.777
13. Schimpff S, Satterlee W, Young VM, Serpick A (1971) Empiric therapy with carbenicillin and gentamicin for febrile patients with cancer and granulocytopenia. N Engl J Med 284 (19):1061-1065

14. Schmitz T, Bair N, Falk M, Levine C (1995) A comparison of five methods of temperature measurement in febrile intensive care patients. Am J Crit Care 4(4):286-292

15. Shellock FG, Rubin SA (1982) Simplified and highly accurate core temperature measurements. Med Prog Technol 8(4):187188 